\title{
Estudio estructural de membranas cyclopore de microfiltración
}

\author{
J. I. CALVO, L. PAlACiO, P. PRÁDANOS, A. MARTíN, F. MARTíneZ Y A. HERNÁNDEZ \\ Dpto. Termodinámica y Física Aplicada, Facultad de Ciencias, Universidad de Valladolid, 47071 Valladolid, España
}

\begin{abstract}
La Microscopía de Fuerza Atómica (AFM), de reciente desarrollo, se ha revelado como una técnica especialmente valiosa en la caracterización de todo tipo de superficies y materiales. En este trabajo se ha utilizado dicha técnica en la caracterización superficial de diversos filtros de microfiltración, obtenidos por el método de revelado de trazas nucleares, y en consecuencia, especialmente útiles como membranas test, debido a su simple estructura. Para estas membranas se han estudiado diversos parámetros de interés estructural, como son la porosidad, la distribución de tamaños de poro y la rugosidad superficial de las mismas. Todos estos parámetros han sido convenientemente analizados y sus resultados comparados con los obtenidos por otras técnicas de caracterización complementarias, a fin de asegurar su validez y fiabilidad. Se ha obtenido información importante sobre la estructura de dichas membranas, especialmente se ha determinado una importante asimetría entre las caras de la membrana, asimetría que se atribuye al proceso de bombardeo que constituye la fase esencial del método de fabricación de las mismas.
\end{abstract}

Palabras clave: Membranas, microfiltración, microscopía de fuerza atómica, porometría de aire, porosimetría de mercurio.

Structural study of cyclopore microfiltration membranes.

Atomic Force Microscopy (AFM), a recently developed technique, has proved to be a specially useful tool to characterise different types of material surfaces. In this work, we have used AFM to achieve a complete surface characterisation of several microfiltration membranes. These membranes are obtained by the track-etching method and, consequently, they are very suitable as test membranes, as having a very simple porous structure. For these membranes, several interesting structural parameters have been studied, as: porosity, pore size distributions and surface roughness of the membrane samples. All these parameters, conveniently analysed, have been compared with results obtained from other complementary characterisation techniques, to assure the realiability and validity of the obtained results. Important information on the membrane samples structure has been obtained. In that sense has been determined a clear asymmetry between both membrane sides, asymmetry that can be attributed to the manufacture process itself.

Keywords: Membranes, microfiltration, atomic force microscopy, air displacement porometry, mercury porosimetry.

\section{INTRODUCCIÓN}

Las membranas de microfiltración presentan un extenso campo de utilización industrial, con aplicaciones plenamente desarrolladas en campos tan diversos como procesos farmacéuticos, biotecnología o producción de alimentos. A fin de optimizar la utilización de los diversos tipos de membranas existentes en el mercado en las diferentes aplicaciones industriales posibles, se hace necesario un completo y amplio conocimiento previo de las características y posibilidades de dichos filtros. En este sentido, su caracterización estructural, tanto superficial como en volumen, se puede acometer utilizando un amplio abanico de técnicas más o menos complementarias, cuyos rangos de aplicación y características específicas no son suficientemente comprendidos (1).

Entre dichas técnicas se pueden destacar, como más frecuentemente usadas, las siguientes:

i) Microscopía electrónica: con la posibilidad de usar diferentes técnicas para observar la superficie o cortes transversales de la membrana. Así se tiene SEM (microscopía electrónica de barrido), TEM (microscopía electrónica de transmisión), FESEM (microscopía electrónica de barrido por emisión campo), etc. Generalmente las imágenes son posteriormente estudiadas mediante análisis computerizado para obtener las distribuciones de tamaño de poros.

ii) Microscopía de fuerza atómica: Esta técnica permite el estudio superficial de materiales no conductores hasta la escala molecular.

iii) Test de punto de burbuja: método introducido por Bechhold, y basado en la medida de la presión necesaria para hacer pasar un fluido a través de una membrana llena de líquido.

iv) Porosimetría de mercurio: método basado en los mismo principios que el de punto de burbuja, aunque en este caso es el mercurio (líquido no-mojante), el que se usa para llenar la membrana seca.

v) Métodos de adsorción-desorción: basados en el uso de la ecuación de Kelvin, que relaciona la presión reducida de vapor de un líquido en una superficie curvada con la misma presión en equilibrio con un plano. Asímismo la teoría BET de adsorción se utiliza frecuentemente para determinar las áreas superficiales específicas.

vi) Permoporometría: técnica basada en el bloqueo controlado de los poros por condensación del vapor presente como componente de una mezcla gaseosa, y la medida simultánea del flujo de gas a través de la membrana. 
vii) Termoporometría: otro método sugerido por Brun y col., basado en el hecho de que el punto de solidificación del vapor condensado en los poros es función de la curvatura de la interfase.

viii) Test de retención de solutos: se miden las retenciones, en condiciones más o menos estandarizadas, para varios solutos de peso molecular o radio hidrodinámico crecientes.

Entre estas técnicas, las que se refieren a sus características superficiales son especialmente relevantes, por cuanto gran parte de la adsorción y ensuciamiento tienen lugar sobre la superficie activa. En este sentido resultan de gran interés las técnicas microscópicas, que permiten obtener directamente distribuciones de tamaños de poro en superficie. Existen dos técnicas de uso más frecuente: TEM y SEM. Recientemente FESEM se ha usado también en el análisis de membranas permitiendo resoluciones de hasta $0.7 \mathrm{~nm}$, utilizando bajos voltajes de aceleración (1).

Pero, incluso en este caso, la microscopía electrónica puede ocasionar daños en la estructura de la membrana difícilmente cuantificables y que falsean los resultados obtenidos. En este sentido, las técnicas microscópicas de sonda han supuesto una revolución, permitiendo resoluciones hasta nivel molecular al tiempo que no producen ningún daño a la muestra observada. Entre ellas, la Microscopía de Fuerza Atómica (AFM) da excelentes prestaciones para materiales no-conductores, como son los habitualmente encontrados en la tecnología de membranas. Desarrollada por Binning y col., (2), su principal ventaja sobre las técnicas de microscopía electrónica es que no se necesita preparación previa de la muestra (3). Aún siendo una técnica relativamente novedosa, ya ha encontrado numerosas aplicaciones en la caracterización de membranas, tanto biológicas como sintéticas $(4,5)$.

Las membranas fabricadas por revelado químico de trazas nucleares son especialmente útiles, debido a su estructura simple, con el fin de ajustar modelos e interpretar los resultados obtenidos mediante las diversas técnicas de caracterización. Debido a la simplicidad de estas membranas, las diversas técnicas de caracterización pueden compararse entre sí de manera sencilla.

\section{PARTE EXPERIMENTAL}

\subsection{Membranas}

En este trabajo se han estudiado membranas Cyclopore ${ }^{\circledR}$, suministradas por Whatman y fabricadas mediante revelado de trazas. Dichas trazas se obtienen por el bombardeo de protones sincrotrónicos, obtenidos de una fuente de radiofrecuencias, sobre láminas de policarbonato. Como resultado se obtie- nen membranas planas que, tras el correspondiente revelado químico, presentan un conjunto de poros que se pueden suponer razonablemente cilíndricos y que atraviesan perpendicularmente la membrana. De esta forma se obtienen, controlando el tiempo de revelado, membranas con diversos tamaños nominales de poro, todos ellos en el rango de Microfiltración. En este caso se han analizado muestras con diámetros nominales de poro de 0.1, 0.2, 0.4, 0.6, 0.8 y $1.0 \mu \mathrm{m}$, a las cuales se denominarán, de aquí en adelante, C01, C02, C04, C06, C08 y C10, respectivamente. Algunos datos nominales de dichas membranas suministrados por el fabricante se muestran en la Tabla I.

\subsection{Equipos y Técnicas Experimentales}

En la obtención de imágenes de las membranas se utilizó un microscopio de fuerza Atómica Nanoscope-IIIA ${ }^{\circledR}$, de Digital Inst. Así se obtuvieron imágenes de diferentes muestras escogidas aleatoriamente de cada tipo de membrana. El área de escaneado se escogió adecuadamente para cada membrana, de forma que se tuviera buena definición en las fronteras de los poros, manteniendo asímismo, dentro del campo de barrido, un número suficiente de poros que asegurará la representatividad del posterior análisis estadístico. Las imágenes se obtuvieron utilizando el modo de contacto intermitente (Tapping Mode $\left.^{\circledR}\right)$, a temperatura ambiente, y mediante unas puntas de nitruro de silicio (NanoTips ${ }^{\circledR}$ ), de una alta relación longitudanchura, de forma que minimicen la posible convolución entre la punta y las entradas de los poros.

Posteriormente se realizó un análisis de imagen computerizado, utilizando un software comercial (Jandel ${ }^{\circledR}$ ScanPro, versión 3.00.0030), de forma que cada microfotografía, digitalizada a una resolución de $1024 \times 768$ pixels, fue convertida en una imagen en niveles de gris. A continuación se realizó una equalización para eliminar los cambios parásitos de nivel de gris y posteriormente el espectro se expandió buscando el máximo contraste y definición, (6). Las imágenes resultantes fueron redefinidas de acuerdo a un nivel umbral de gris asignando los niveles superiores a los poros y el resto a la matriz sólida de la muestra. Evidentemente la etapa de selección del umbral resulta fundamental para realizar un correcto análisis de los poros así asignados. En cualquier caso todo procedimiento razonable de selección debe ser auxiliado por una inspección visual de las imágenes que permita asegurar la bondad del criterio elegido.

TABLA I. DATOS NOMINALES DEL FABRICANTE PARA LOS 6 FILTROS CYCLOPORE USADOS.

(1) VELOCIDAD INICIAL DE FLUJO POR UNIDAD DE ÁREA DE MEMBRANA CON AGUA PREFILTRADA A $25^{\circ} \mathrm{C}$ y 0.7 x $10^{5} \mathrm{~Pa}$.

(2) Velocidad INICIAL DE FLUJO POR UNIDAD DE ÁREA DE MEMBRANA CON AIRE PREFILTRAdO A $25^{\circ} \mathrm{C}$ y 0.7 x $10^{5} \mathrm{~Pa}$.

\begin{tabular}{|c|c|c|c|c|c|c|c|c|}
\hline Membrana & $\begin{array}{c}\text { Diámetro } \\
(\mu \mathrm{m})\end{array}$ & $\begin{array}{l}\text { Espesor } \\
(\mu \mathrm{m})\end{array}$ & $\begin{array}{c}\text { Densidad } \\
\text { de Poros } \\
\left(10^{12} \text { Poros } m \mathbf{m}^{2}\right)\end{array}$ & $\begin{array}{c}\text { Porosidad } \\
\text { Media } \\
(\%)\end{array}$ & $\begin{array}{c}\text { Punto de Burbuja } \\
\text { en Agua } \\
\text { (10 Pa) }\end{array}$ & $\begin{array}{c}\text { Flujo Típico } \\
\text { en Aga }{ }^{1} \\
\left(1 / \mathrm{s} \cdot \mathrm{m}^{2}\right)\end{array}$ & $\begin{array}{c}\text { Flujo Típico } \\
\text { en Aire } \\
\left(10^{q} \mathrm{~s}_{\mathrm{s}} \mathrm{m}^{2}\right)\end{array}$ & $\begin{array}{c}\text { Fuerca Típica } \\
\text { de Rotura } \\
\left(10^{5} \mathrm{~Pa}\right)\end{array}$ \\
\hline $\mathrm{COl}$ & 0.1 & 10 & 6.0 & 4 & $>6.9$ & 0.50 & 2.17 & $>1.38$ \\
\hline $\mathrm{CO} 2$ & 0.2 & 10 & 4.5 & 14 & 5.1 & 4.00 & 10.83 & $>0.69$ \\
\hline $\mathrm{CO} 4$ & 0.4 & 10 & 1.0 & 13 & 2.5 & 12.17 & 20.83 & $>1.03$ \\
\hline $\mathrm{COS}$ & 0.6 & 9 & 0.3 & 8 & 1.8 & 14.67 & 20.83 & $>2.07$ \\
\hline $\mathrm{CO} 8$ & 0.8 & 9 & 0.3 & 15 & 1.1 & 33.33 & 36.67 & $>2.07$ \\
\hline $\mathrm{Cl0}$ & 1.0 & 9 & 0.2 & 16 & 0.95 & 36.67 & 33.33 & $>2.07$ \\
\hline
\end{tabular}


Los datos obtenidos del análisis de las imágenes de AFM fueron comparados con resultados provenientes de otras técnicas de caracterización habituales en el análisis de membranas y materiales porosos en general. Así se utilizó un equipo de adsorción-desorción de gases (con permeación de helio), un Omnisorp 100-CX de Coulter Ltd., para determinar la porosidad de las membranas mediante una técnica desarrollada en este grupo, (7).

Por otro lado se utilizó un porosímetro de mercurio, Autopore-III de Micromeritics, con el cual se determinaron las distribuciones de tamaños de poro así como la porosidad. Finalmente, un porómetro de aire, Coulter Porometer II, de Coulter Ltd., que utiliza la técnica de desplazamiento de líquidos, (8), permitió determinar la distribución de tamaños de los poros abiertos al flujo.

El uso conjunto de todas estas técnicas permitió determinar los siguientes parámetros de interés en la caracterización de estos filtros:

Porosidades: tanto en volumen, mediante volumetría de permeación de helio y porosimetría de mercurio; como en superficie, a través de los datos de análisis de las imágenes de AFM.

Distribución de tamaño de poros en volumen, mediante: porosimetría de mercurio que analiza todos los poros (abiertos o no) presentes en el volumen de la membrana; y porometría de aire-líquido que detecta únicamente los poros activos (abiertos al flujo).

Distribución de tamaño de poros en superficie, mediante microscopía de fuerza atómica (AFM), estudio realizado en ambas caras de la membrana.

Rugosidades superficiales, mediante microscopía de fuerza atómica (AFM), asímismo en ambas caras de la membrana.

\section{RESULTADOS}

En la Figura 1 se tienen sendos ejemplos de imágenes de la superficie de una membrana Cyclopore. Las imágenes representan las dos caras de una muestra de la membrana C10. Se aprecia claramente que existe una evidente asimetría entre ambas caras de la membrana, con una de las caras (a la que se denominará brillante en lo que sigue) mucho más pulida, donde se destacan nítidamente los orificios que dan lugar a los poros. Por otro lado, la otra cara de la membrana (que denominaremos mate) tiene mucha mayor rugosidad y, junto a los poros reales, presenta una serie de huecos de difícil interpretación. Es importante comentar que el fabricante considera estos filtros como simétricos, suponiendo iguales prestaciones por ambos lados de la membrana.

De todas las membranas se midieron, mediante el correspondiente software de análisis de imagen, las distribuciones de tamaños de poros, tanto por la cara brillante como por la mate. Ejemplos de las distribuciones obtenidas se presentan en las Figuras 2 y 3, para las caras brillante y mate, respectivamente, de la membrana C04. Se observa claramente en estas figuras, así como en las correspondientes al resto de membranas, como las distribuciones de tamaños obtenidas son relativamente estrechas y se pueden asimilar razonablemente a distribuciones gaussianas. Se han ajustado dichas distribuciones a una distribución normal, a fin de obtener los diámetros medios de poro y las desviaciones estándar. Los resultados de dichos ajustes, para todas las membranas analizadas, se presentan en la Tabla II.

Se observa como estos datos se acercan razonablemente a los valores nominales, con mejor acuerdo para los resultados de la

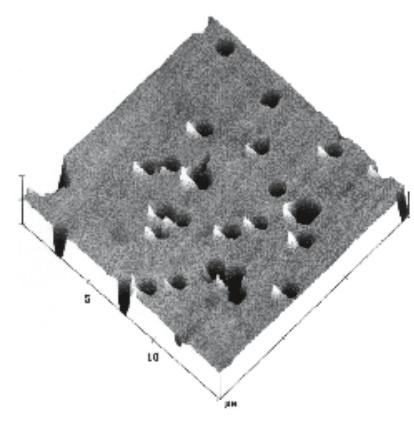

C10 - Brillante

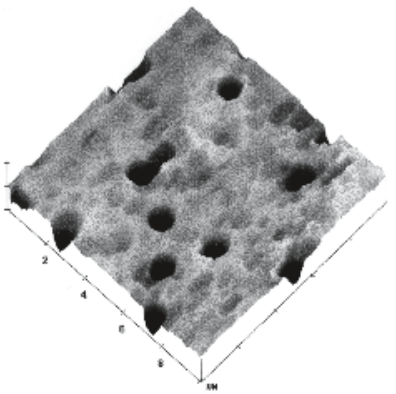

C10 - Mate
Fig. 1.- Imágenes 3D de AFM de la superficie de la membrana C10, por su cara brillante y mate.

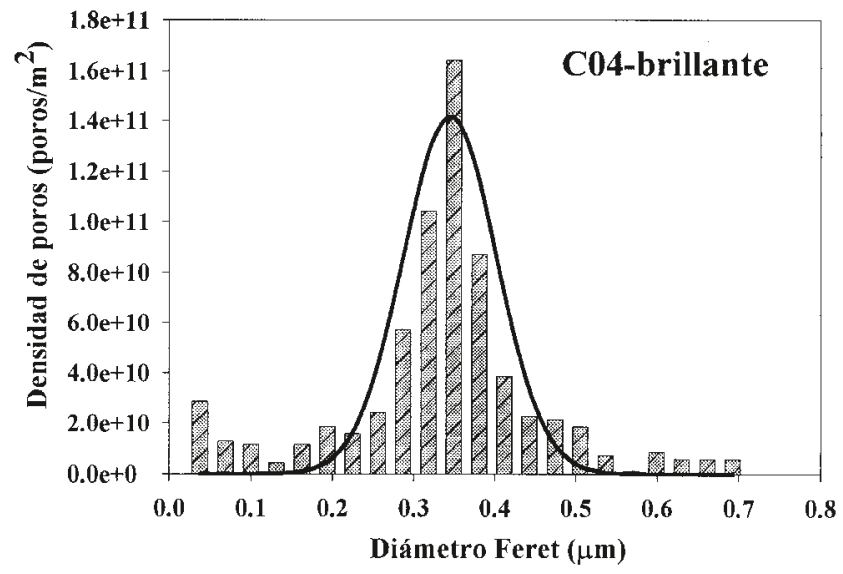

Fig. 2.- Distribución de tamaños de poros de la cara brillante de la membrana C04.

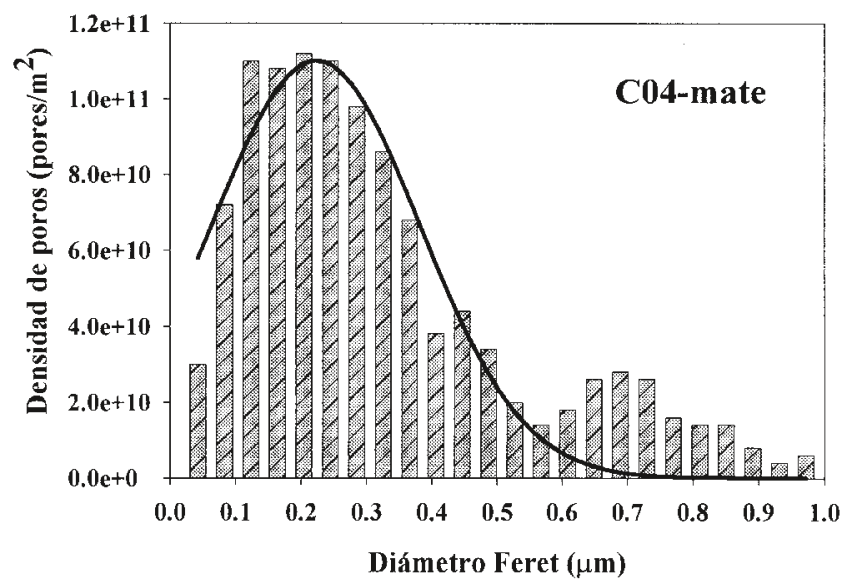

Fig. 3.- Distribución de tamaños de poros de la cara mate de la membrana C04. 


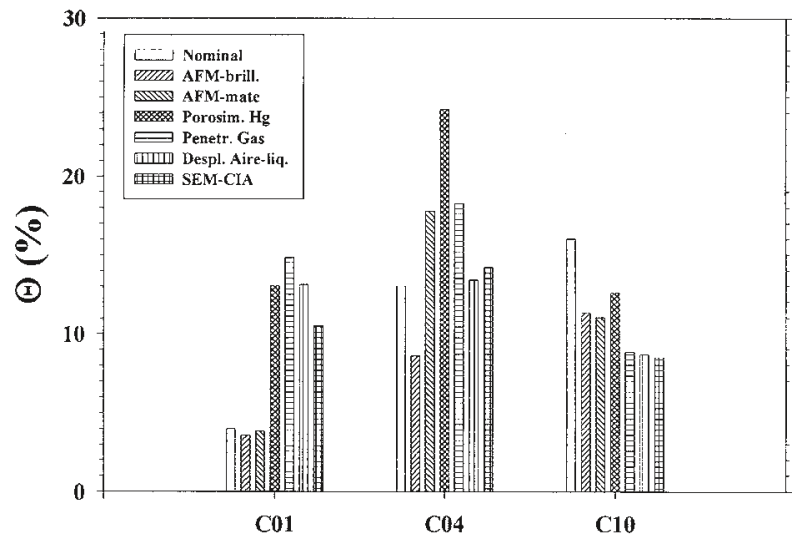

Membranas

Fig. 4.- Resultados de la porosidad según diversas técnicas de caracterización.

cara brillante, excepto para la membrana de tamaño de poro más pequeño (la C01) cuyo diámetro de poro nominal es más acorde con el dato obtenido de las caras mates.

Los resultados obtenidos por Microscopía de Fuerza Atómica se han comparado, asímismo en la Tabla II, con resultados de Porometría de Desplazamiento de Líquidos y de Porosimetría de Mercurio. Se observa un buen acuerdo entre los resultados procedentes de las diversas técnicas de caracterización. Este acuerdo va siendo mayor cuanto mayor es el diámetro de poro nominal. La razón puede ser debida a la mayor definición de los bordes de los poros que se tienen cuanto mayor es el tamaño de éstos, lo cual conlleva la minimización del error de definición.

Por otro lado, las técnicas con las que se comparan los diámetros de poro cubren aspectos diferentes con lo que, en principio no es esperable un total acuerdo entre datos obtenidos por dichas técnicas. Así la Microscopía de Fuerza Atómica da información sobre la superficie de los poros mientras que las otras dos técnicas informan sobre los poros activos (i.e., aquellos que atraviesan completamente la membrana, contribuyendo realmente al flujo) e incluso de poros internos que no llegan a contribuir a las características funcionales y selectivas de estos filtros (como ocurre en la porosimetría de mercurio).

Siguiendo con el análisis de los resultados de AFM, se obtuvieron las porosidades superficiales y números totales de poros en ambas caras de la membrana. En la Tabla III, se presentan dichos datos para ambas caras. Se observa nuevamente un

TABLA II.- DiÁMETROS DE PORO MEDIO (EN mm) DE LAS MEMBRANAS CYCLOPORE, SEGÚN DIVERSOS MÉTODOS DE CARACTERIZACIÓN.

\begin{tabular}{|c|c|c|c|c|}
\hline Membrana & $\begin{array}{c}\text { AFM } \\
\text { (cara brillante) }\end{array}$ & $\begin{array}{c}\text { AFM } \\
\text { (cara mate) }\end{array}$ & $\begin{array}{c}\text { Desplazamiento } \\
\text { de Aire-líquido }\end{array}$ & $\begin{array}{c}\text { Porosimetría } \\
\text { de Mercurio }\end{array}$ \\
\hline C01 & 0.069 & 0.082 & 0.135 & 0.13 \\
C02 & 0.13 & 0.1171 & 0.265 & - \\
C04 & 0.35 & 0.223 & 0.462 & 0.36 \\
C06 & 0.47 & 0.38 & 0.680 & - \\
C08 & 0.710 & 0.694 & 0.988 & - \\
C10 & 0.99 & 0.72 & 1.162 & 0.5 \\
\hline
\end{tabular}

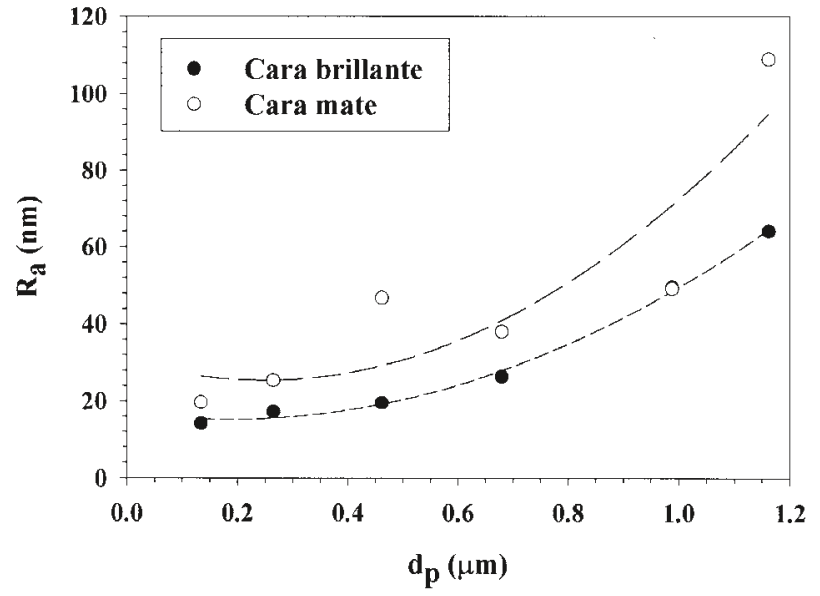

Fig. 5.-Rugosidad de las membranas Cyclopore frente al diámetro de poro medio obtenido mediante Desplazamiento de Aire-líquido, tanto para la cara brillante como mate.

acuerdo razonable con los valores nominales propuestos por el fabricante, aunque en algunos casos las desviaciones son importantes, especialmente respecto a los datos de porosidad. Estos valores de la porosidad se han comparado con los procedentes de diversas técnicas de caracterización, $(7,9,10)$. Los resultados de dicha comparación se presentan en la Figura 4, para un conjunto representativo de las membranas analizadas.

En principio cabría esperar que los métodos de caracterización intrusivos como son porosimetría de mercurio y penetración de gases, nos llevaran a los mismos o similares valores. Ello no es así, dado que los bajos volúmenes porosos que presentan estas membranas conllevan un alto error experimental en el caso de la porosimetría de mercurio, errores experimentales difíciles de soslayar, unidos al efecto de compresión del material a las altas presiones aplicadas. En cuanto a los valores obtenidos por AFM, se acercan al resto de los valores a medida que va creciendo el tamaño de poro nominal, siguiendo la pauta encontrada en los diámetros de poro medios.

Finalmente se han estudiado los valores de la rugosidad de las membranas, valores que igualmente se han determinado en ambas caras de la misma. A tal fin se ha considerado, como parámetro más significativo, la rugosidad promedio que nos da cuenta de las fluctuaciones de altura que experimenta la punta cuando recorre la superficie de la muestra. Los datos de rugosidad se han representado en la Figura 5, frente al diámetro de poro medio obtenido por porosimetría de desplazamiento de líquidos. Así las membranas con mayores tamaños
TABLA III.- $\quad$ DENSIDADES DE POROS Y POROSIDADES OBTENIDAS POR ANÁLISIS DE LAS IMÁGENES AFM DE LAS CARAS BRILLANTE Y MATE.

\begin{tabular}{|c|c|c|c|c|}
\hline \multirow[t]{2}{*}{ Membrana } & \multicolumn{2}{|c|}{ Cara brillante } & \multicolumn{2}{|c|}{ Cara mate } \\
\hline & $\begin{array}{c}\mathrm{N} \\
\left(10^{12} \text { poros } / \mathrm{m}^{2}\right)\end{array}$ & $\begin{array}{c}\Theta \\
(\%)\end{array}$ & $\begin{array}{c}\mathrm{N} \\
\left(10^{12} \cdot \text { poros } / \mathrm{m}^{2}\right)\end{array}$ & $\begin{array}{c}\Theta \\
(\%)\end{array}$ \\
\hline $\mathrm{C} 01$ & 4.73 & 3.56 & 4.96 & 3.87 \\
\hline $\mathrm{C} 02$ & 3.84 & 5.94 & 3.94 & 5.84 \\
\hline $\mathrm{C} 04$ & 0.643 & 8.6 & 1.14 & 17.78 \\
\hline $\mathrm{C} 06$ & 0.366 & 6.42 & 0.634 & 11.81 \\
\hline $\mathrm{C} 08$ & 0.205 & 8.49 & 0.186 & 7.06 \\
\hline $\mathrm{C} 10$ & 0.106 & 11.33 & 0.198 & 11.03 \\
\hline
\end{tabular}


de poro presentan rugosidades igualmente mayores. Este hecho puede ser asignado a la cercana relación existente entre poros y rugosidad, de forma que los poros producen la mayor parte de la contribución detectada en la rugosidad.

Por otro lado, se observa nuevamente la clara diferencia entre ambas caras de la membrana, con rugosidades generalmente mucho mayores en la cara mate, consecuencia de los huecos que se observaban en las figuras topográficas (ver Figura 1.b). Asímismo, las rugosidades de la cara mate no presentan una relación tan clara con el tamaño de poro medio como los datos obtenidos en la cara brillante, hecho sin duda debido a la mayor aleatoriedad de los defectos presentes en las caras mates.

\section{CONCLUSIONES}

El estudio realizado por AFM, ha permitido comprobar la asimetría existente entre ambas caras de la membrana: presentando una de las caras, junto a un aspecto más brillante (consecuencia posiblemente del proceso industrial de pulido), unos poros más definidos, regulares en tamaño y forma (casi perfectamente circular), en comparación con la cara mate.

Esta asimetría se ha constatado asímismo en las distribuciones de tamaños de poro obtenidas de estas imágenes, con distribuciones generalmente más estrechas y gaussianas en el caso de la cara brillante. Por otro lado, la comparación entre los resultados de tamaño de poro medio obtenidos por las diferentes técnicas, aún respondiendo a diferentes características, nos da importantes conclusiones:

- Los datos de AFM presentan generalmente mejor coincidencia en el caso de la cara brillante, con otras técnicas de caracterización más internas. En este sentido se puede afirmar que la cara brillante representa los poros realmente existentes en la membrana, mientras que los datos de la cara mate están enmascarados por una fuerte presencia de defectos que se atribuiría a trazas dejadas por partículas sin suficiente energía para atravesar totalmente la matriz sólida de la membrana (no dando lugar, consecuentemente, a poros reales).

- Por otro lado el acuerdo razonable entre datos superficiales (obtenidos por AFM) y datos internos (p.ej., los de desplazamiento de líquidos), indica que estas membranas responden muy cercanamente al modelo supuesto de poros cilíndricos perpendiculares a la superficie de la membrana.

El resto de los resultados obtenidos (número de poros y porosidad) dan cuenta asímismo de la validez de las conclusiones esbozadas, con porosidades y densidades de poros generalmente mayores en la cara mate (especialmente en aquellas membranas, $\mathrm{C} 04$ y C06, donde las supuestas cavernas o imperfecciones contribuyen más a las distribuciones totales).
Finalmente el análisis de las rugosidades permite concluir, con más claridad en el caso de las caras brillantes, que dicho parámetro está fuertemente relacionado con el tamaño de los poros, creciendo con éstos. Esto es, en principio, bastante razonable, si se tiene en cuenta que en los poros más grandes la punta del microscopio de fuerzas puede penetrar más profundamente, contribuyendo apreciablemente al aumento de la rugosidad.

En resumen, la Microscopía de Fuerza Atómica presenta interesantes posibilidades en el análisis de la superficie de materiales porosos, dando información que debidamente complementada con otras técnicas de caracterización, permite obtener un conocimiento indudablemente preciso de la estructura completa de la membrana.

\section{AGRADECIMIENTOS}

Los autores desean agradecer la financiación de este trabajo a través del proyecto QUI96-0767 del Plan Nacional de I+D.

\section{BIBLIOGRAFÍA}

1.- A. Hernández, J.I. Calvo, P. Prádanos and L. Palacio, A Multidisciplinary Approach Towards Pore Size Distributions in Microporous and Mesoporous Membranes, en Surface Chemistry and Electrochemistry of Membranes (Surfactant Science Series /79), Ed. T.S. Sorensen, Marcel Dekker Inc., Nueva York, EE.UU. (1999).

2.- G. Binnig, C.F. Quate and Ch. Gerber, Atomic force microscopy, Phys. Rev. Lett., 56, 930-933 (1986)

3.- S. Nakao, Determination of pore size and pore size distribuion. 3. Filtration membranes, J. Membrane Sci, 96 131-165. (1994)

4.- A. K. Fritzsche, A.R. Arevalo, M.D. Moore, C.J. Weber, V.B. Elings, K. Kjolle and C.M. Wu, The surface structure and morphology of polyvinylidene fluoride microfiltration membranes by atomic force microscopy, J. Membrane Sci, 68 65-78. (1992)

5.- P. Dietz, P.K. Hansma, O. Inacker, H.D. Lehmann and K.H. Herrmann, Surface pore structures of micro- and ultrafiltration membranes imaged with the atomic force microscope, J. Membrane Sci., 65 101-111. (1992).

6.- A. Hernández, J.I. Calvo, P. Prádanos, L. Palacio, M ${ }^{a}$ L. Rodríguez y J.A. de Saja, Surface structure of microporous membranes by computerized SEM image analysis applied to Anopore filters, J. Colloid \& Interface Sci., 137, 89-97. (1997).

7.- L. Palacio, P. Prádanos, J.I. Calvo y A. Hernández, Porosity measurements by a gas penetration method and other techniques applied to membrane characterization, Thin Solid Films, 348, 22-29, (1999).

8.- A. Hernández, J.I. Calvo, P. Prádanos, y F. Tejerina, Pore size distributions in microporous membranes. A critical analysis of the bubble point extended method, J. Membrane Sci., 112, 1-12. (1996)

9.- J.I. Calvo, A. Hernández, G. Caruana y L. Martínez, Pore size distributions in microporous membranes. I. Surface study of track-etched filters by image analysis, J. Colloid \& Interface Sci., 175, 138-150. (1995).

10.-L. Palacio, Caracterización estructural y superficial de membranas microporosas, Tesis doctoral, Universidad de Valladolid, Valladolid, 1998. 